\title{
RESTAURACIÓN DE ZONAS NATURALES ALTERADAS POR ACTIVIDADES MINERAS A CIELO ABIERTO
}

\author{
(THE RESTORATION OF LAND AFFECTED BY OPEN-CAST MINING)
}

\author{
M. ${ }^{a}$ Paz Aramburu, M. ${ }^{a}$ del Milagro Escribano y Mercedes de Frutos \\ Departamento de Proyectos y Planificación Rural. \\ Universidad Politécnica de Madrid.
}

\section{RESUMEN}

El principal objetivo en la restauración de tierras afectadas por actividades mineras es minimizar el impacto en el área circundante, de manera que la zona afectada recupere su uso original y se reintegre en su entorno.

En este artículo se describen algunos de los criterios a aplicar en la remodelación de escombreras y taludes finales de frentes de corta, con objeto de conseguir la integración de estos elementos en el paisaje circundante.

Los factores que intervienen en la configuración de los terrenos alterados son muy diversos, unos de carácter económico, otros topográficos y otros estéticos.

La remodelación va a condicionar otras facetas de la recuperación como son la evolución del suelo y el establecimiento de la vegetación.

Todos estos condicionantes obligan a considerar el modelado del terreno integrado en el plan de restauración y de forma simultánea a la explotación para evitar futuros impactos o minimizar los ya existentes.

\section{SUMMARY}

The main reason for restoring lands affected by mining operations is to minimize the impact on the surrounding area, in such a way that the affected zone is reintegrated into the environment, with the land being put to its original use.

A description of the generic criteria to be applied as regards the shape of slag heaps and final cutting fronts and slopes, in order for them to blend in with the surrounding landscape, is given below.

Design of the final configuration of the terrain following restoration is conditioned by a series of factors that may be grouped under three overall headings: topographic, aesthetical and economical.

It should also be taken into account that the remodelling will impact on other facets of the process of recuperation, such as the evolution of the soil and the establishment of vegetation.

All of the above conditioning factors mean that consideration of the modelling of the terrain must be integrated into the overall planning of the restoration process (even during the initial stages), and that the technical and economic feasibility of the project requires that this be done simultaneously with explotation.

\section{INTRODUCCIÓN}

La tierra es el soporte sobre el cual el ser humano desarrolla todas sus actividades, con la intención de cambiarla y dirigirla hacia situaciones más directamente aprovechables y económicamente favorables. Este hecho aparece como una constante que se repite desde los albores de la civilización. Sin embargo, hasta hace algo más de un siglo, la gran mayoría de las agresio. nes ambientales eran absorbidas por la acción regeneradora de la naturaleza, al ser muy numerosos los recursos naturales existentes en comparación con la escasa, aunque creciente, actividad transformadora del hombre.
En la actualidad los cambios y modificaciones que se producen en el medio natural adquieren dimensiones alarmantes, no solamente al aumentar la demanda de recursos naturales sin experimentar el mismo incremento las reservas, sino también por la alteración del equilibrio ecológico que esas acciones producen.

Por otra parte, y paradógicamente, la escasez de recursos va acompañada del abandono de cultivos en muchas zonas que han dejado de ser productivas, medidas con el rasero de los actuales parámetros económicos. A esta cuestión debe añadirse el proceso de desertización y las acciones de deforestación que al alcanzar dimensiones territoriales muy grandes no en- 


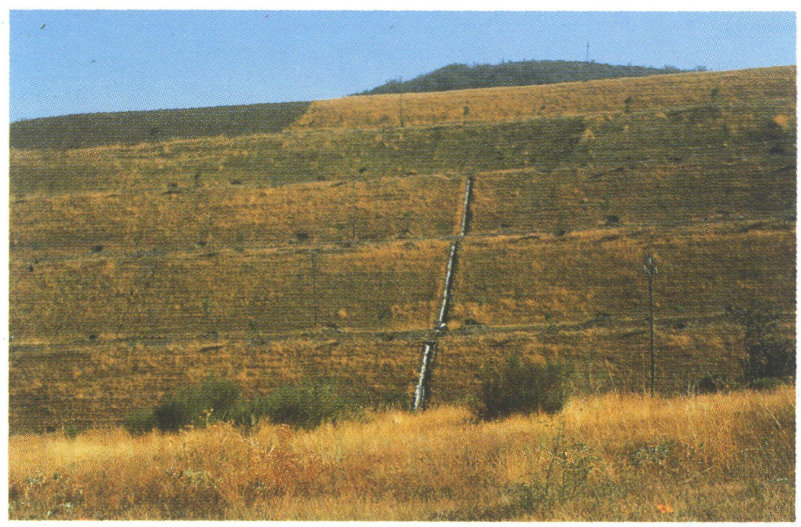

Las labores de recuperación llevadas a cabo en algunas escombre ras no siempre son las más adecuadas como puede observarse en esta foto correspondiente a una explotación de carbón situada en Sabero (León).

La forma excesivamente regular, la disposición geométrica de las bermas y bajantes, la angulosidad de las uniones berma-talud, etc., producen un impacto visual que dificulta la integración de la escombrera en el entorno natural.

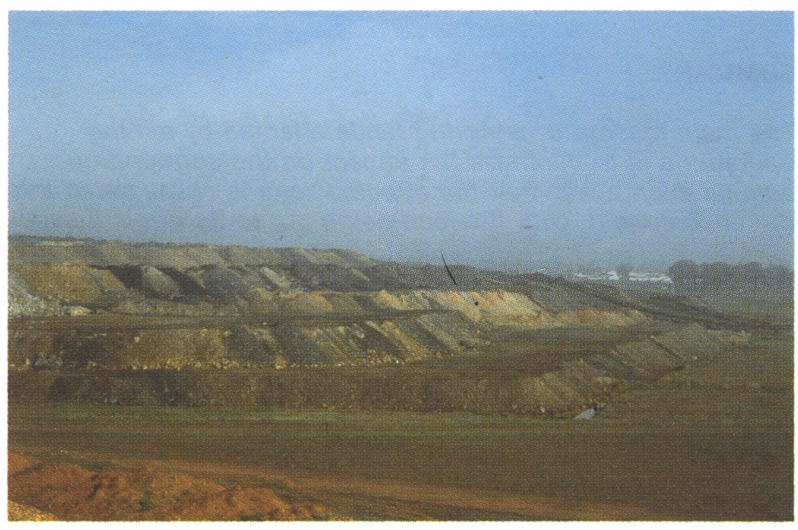

Aspecto que presentan las escombreras originadas en la minería de carbón a cielo abierto en el Valle del Guadiato (Córdoba) causantes de un fuerte impacto en el medio circundante ya que constituyen un elemento destacable en la llanura y presentan un elevado contraste debido a su volumen, forma, color y líneas rectas.

tran a formar parte de lo que suele entenderse como recuperación de tierras, concepto éste que se ciñe fundamentalmente al ámbito de ciertas actividades indus. triales.

Este es el caso de la construcción de redes de accesos (carreteras, ferrocarriles...), las actividades extractivas, etc., que han contribuido y contribuyen cada día más a la transformación generalizada del medio biofísico, que es explotado con demasiada frecuencia con una visión a corto plazo, sin considerar el carácter irreversible de muchas de las alteraciones que se producen por un uso malentendido.

Es preciso pues considerar la explotación de la tierra con una visión de futuro en el marco de la ordenación del territorio, contemplando las actividades que alteran el terreno como usos transitorios y no terminales. Hay que estar preparados para restaurar la capacidad

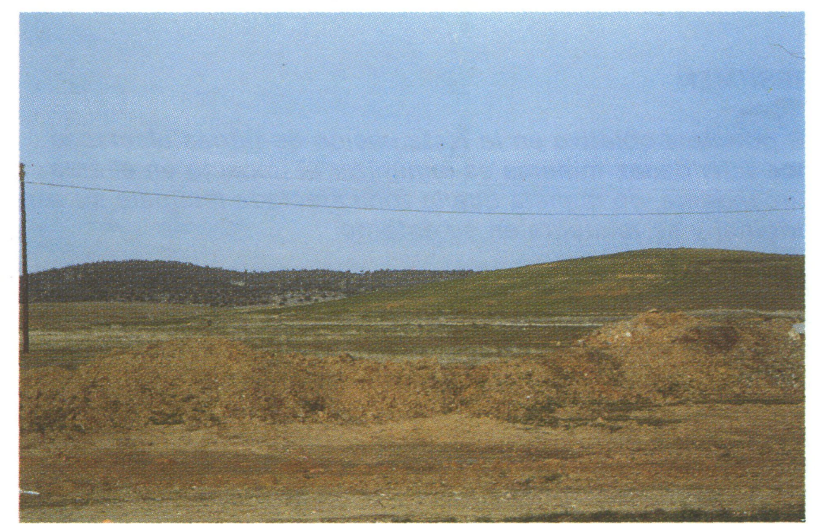

Estado actual de las escombreras en el Valle del Guadiato una vez efectuada la restauración. El trabajo de restauración es correcto tanto desde el punto de vista paisajístico, como de la revegetacion (siembra de cebada) ambos acorde con el entorno de la zona donde están ubicadas.

productiva original de los terrenos sobre los que se actúa. Esto implica una planificación a priori del proceso de recuperación de forma simultánea a la elaboración de los planes de la actividad, es decir, en la fase de diseño y elaboración del proyecto.

En las explotaciones mineras a cielo abierto, actividad protagonista de este artículo, el alto grado de mecanización conseguido permite una notable elevación de los rendimientos que se traduce en un gran incremento del volumen de tierras removidas, originando huecos debido a las tierras industrialmente no utilizables y que necesariamente deben ser retiradas para alcanzar el filón mineral buscado, y escombreras producidas por el asentamiento de las enormes acumulaciones de estériles que se depositan en los alrededores de la explotación como solución menos desfavorable desde el punto de vista económico, pero que muy frecuentemente son localizaciones fácilmente observa- 
bles desde amplias zonas. Esta situación, como contrapartida, es la causa de una clara agresión físicobiológica al medio en que se insertan, llegando a producir, de no tomarse las medidas oportunas, graves alteraciones en el paisaje y en el equilibrio natural existente.

Recuperación, restauración o rehabilitación son palabras que, aunque etimológicamente tengan diferentes significados, se emplean indistintamente para describir los procesos con los que se pretende mejorar tierras degradadas 0 alteradas. La forma e intensidad del proceso de restauración dependerá del tipo de actuación que se haya ejercido sobre el medio natural provocando problemas de excesiva compactación por el uso de maquinaria pesada, grandes acumulaciones de materiales estériles, ausencia de materia orgánica y nutrientes, inestabilidad de las capas superficiales, etc. El principio general de una restauración debe ser recuperar la calidad original del medio teniendo presente la interdependencia que existe entre vegetación, suelo, clima, fauna y hombre. Su completa interacción define el ecosistema autocontrolado que se pretende alcanzar.

Las explotaciones mineras a cielo abierto son, como ya se ha comentado, una de las actividades producidas por el hombre que mayores efectos negativos pueden provocar sobre el medio natural. Por ello, si no se realiza una restauración del terreno afectado, es poco probable que en un período de tiempo razonablemente corto y de forma natural la zona se integre en el entorno circundante o pueda mantener algún tipo de uso.

Desde septiembre de 1988, fecha en que se aprobó el Reglamento del Real Decreto Legislativo 1302/1986 de 28 de junio, es necesario efectuar un estudio de Impacto Ambiental previo a la implantación de nuevas explotaciones a cielo abierto de minerales. Uno de los puntos que debe constar en dichos estudios es el Plan de Restauración del espacio natural afectado por la actividad, de manera que se proteja el medio biofísico y se integre en el entorno circundante una vez finalizada ésta. Para la aprobación de los planes de restauración se aplicarán los Reales Decretos de 15 de octubre de 1982 y de 9 de mayo de 1984, y demás normas complementarias en la medida que no se opongan a lo establecido por la normativa antes mencionada.

El proyecto de recuperación debe formar parte de la planificación de la actividad y realizarse simultáneamente a los planes de diseño y operaciones de ésta.

Los trabajos de restauración se basan fundamentalmente en el remodelado de formas y la instauración de una cubierta vegetal. Para lograr que se instaure la vegetación es imprescindible crear y preparar un suelo capaz de acoger las especies elegidas para cada caso.

\section{CRITERIOS PARA EL DISEÑO GENÉRICO DE FORMAS}

En el diseño de la configuración de terrenos alterados intervienen factores de muy diversos tipos, unos de carácter económico, otros topográficos y otros estéticos que van a condicionar el proyecto de recuperación.

Los principios generales que han de tenerse en cuenta son:

- Adecuar en lo posible la topografía final a la existente en la zona antes de la actuación, y utilizar cuando sea factible los estériles. para rellenar huecos y adaptarse a las sinuosidades del relieve.

- Intentar reproducir las formas características del paisaje natural del área donde se ubica la explotación, y evitar la introducción de elementos que denoten artificialidad (líneas rectas, ángulos muy marcados, regularidad de formas geométricas, simetrías, etcétera).

- Evitar la colocación de elementos de tamaño desproporcionado respecto a los que definen el paisaje de la zona, respetar la escala.

- Estudiar las características visuales del territorio con el fin de:

- Ocultar o alejar los elementos impactantes, especialmente de los puntos principales de observación.

- Utilizar el cerramiento visual natural como elemento que sirva de soporte o apoyo "visual" de los elementos impactantes, de modo que éstos no supongan una discontinuidad en el terreno natural y que no sobrepasen la línea del horizonte.

- No disminuir el tamaño de la cuenca visual preexistente, introduciendo elementos que por su tamaño o emplazamiento limiten perspectivas.

La remodelación va a condicionar otras facetas de la recuperación, como son la evolución del suelo y el establecimiento de la vegetación, y por tanto debe adaptarse a los requerimientos que exigen los usos del suelo previstos para cada zona.

Todos estos condicionantes obligan a considerar el modelado del terreno integrado en el plan de restauración y de forma simultánea a la explotación para evitar futuros impactos o minimizar los ya existentes.

A continuación se dan algunas recomendaciones para la restauración de las escombreras y frentes de cortas originados en las explotaciones mineras a cielo abierto. 


\section{Escombreras}

Las escombreras y vertederos producidos por la minería a cielo abierto constituyen uno de los elementos de mayor intrusión en el entorno: provocan cambios en la fisiografía del lugar y muros visuales con la consiguiente pérdida de perspectiva. Para conseguir la integración paisajística y minimizar en lo posible las alteraciones visuales y ecológicas que producen, es necesario estudiar una serie de características que ayudarán a conseguir dicha integración:

- La localización geográfica, haciendo referencia en este punto a las características visuales y calidad del paisaje del entorno donde se sitúan las escombreras, así como a la disposición espacial de ésta (localización dispersa, localización única cercana a núcleo urbano, etcétera).

- La situación topográfica, estudia sobre qué unidad fisiográfica se asienta la escombrera (sobre un fondo de valle, sobre ladera, en un páramo, etcétera).

- Los caracteres geométricos: tamaño y forma de la escombrera.

- El material de la escombrera, importante tanto por su influencia en las características visuales (color de los materiales), como en los procesos de erosión (tamaño y composición de los materiales) y establecimiento de la vegetación (calidad de los materiales).

- Estado actual de la escombrera en cuanto al grado de cubierta vegetal, si tuviera, y a la erosión que se hubiera producido.

De todas estas características, son los caracteres geométricos y la situación topográfica (este factor muchas veces con limitaciones), las variables sobre las que se puede incidir principalmente para realizar un diseño integrador.

En cuanto al tamaño cabe decir, que a igualdad del resto de factores, la integración paisajistica será tanto más sencilla y menos costosa cuanto menor sea el volumen de los estériles. Si dicho volumen es considerable, se puede practicar, como criterio preliminar de diseño una minería de transferencia (carbón) o rellenar huecos de antiguas explotaciones cercanas (minería no metálica, canteras).

Se puede proceder a la remodelación de una escombrera reduciendo su altura y repartiendo su volumen sobre una superficie mayor, debiendo ser retirada la tierra vegetal de la nueva superficie a ocupar de forma que luego pueda extenderse sobre la escombrera remodelada, como se ve en la figura 1.

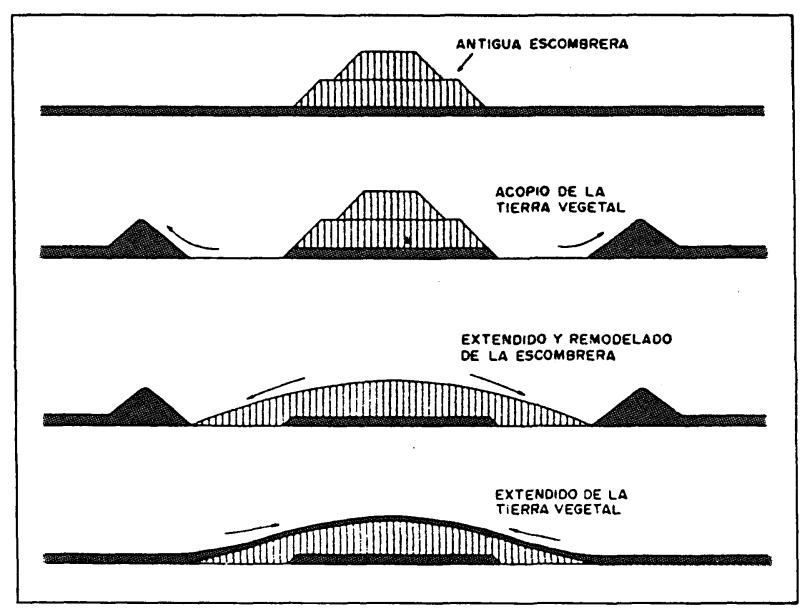

Fig. 1.-Fases a seguir en la remodelación y cobertura con tierra vegetal adyacente de una antigua escombrera (Bradshaw, 1980).

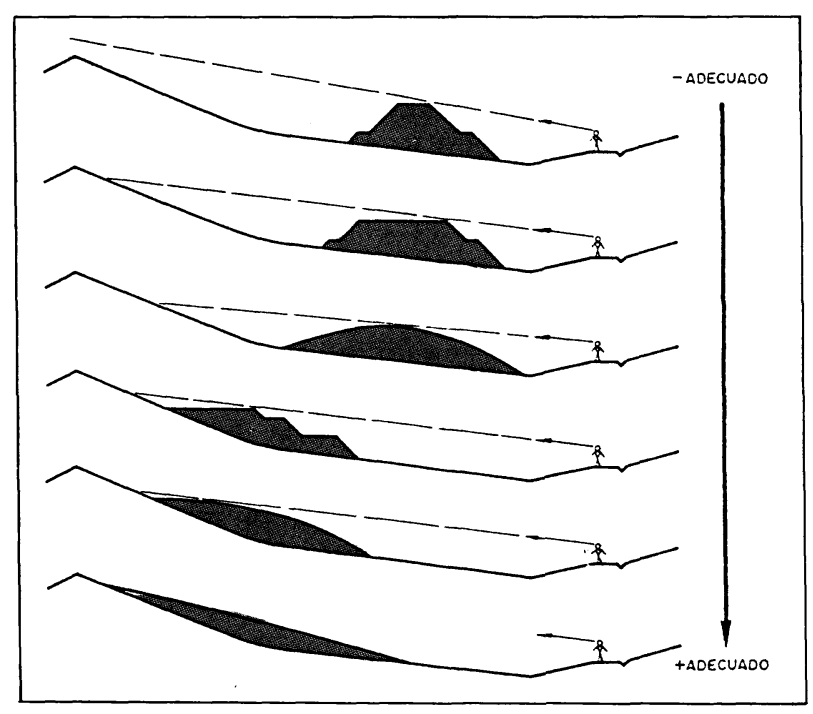

Fig. 2.-Construcción geométrica de un vertedero teniendo en cuenta la fisiografía del entorno.

Esta figura es también un ejemplo de falta de previsión y planificación. En primer lugar, se tapa y queda inutilizable para su uso posterior la tierra vegetal o material de cobertera, que por otra parte es necesario retirar para asegurar la estabilidad del vertedero en situaciones de pendiente. $Y$ en segundo lugar, la remodelación así planteada, es una actuación más cara y laboriosa que si se hubiese diseñado la forma final y se hubiese ido construyendo desde el principio de acuerdo con ella.

En la ubicación de los vertederos se tendrán en cuenta los criterios básicos apuntados: ocultación, aprovechando obstáculos naturales del terreno; alejamiento de los focos principales de observación (carreteras, pueblos,...); no tapar vistas panorámicas, etc. En los terrenos ondulados y montañosos la intrusión visual disminuye si las escombreras se apoyan en las laderas (Figura 2), lo cual puede ser considerado un buen empla- 
zamiento siempre y cuando no lleguen a cegarse los valles o a taponar las líneas principales de drenaje superficial natural.

Otra técnica para la ocultación o enmascaramiento de escombreras es la creación de pantallas. Las escombreras de menor tamaño o una pequeña proporción de estériles, pueden en ocasiones ser utilizados, si se emplazan y diseñan adecuadamente, como pantallas que dificulten la observación de elementos visualmente desfavorables (grandes escombreras, hueco, infraestructura, etc).

Otras recomendaciones ya mencionadas en los criterios generales serían:

- Evitar que la altura de la escombrera sobrepase la cota altitudinal del entorno para que así no destaque en la línea del horizonte, tendiendo siempre un cerramiento visual más apto para absorberla e integrarla (Figura 2).

- Tener en cuenta la distancia de la escombrera a los focos principales de observación, pues distancia y tamaño de la escombrera están directamente relacionados.

En cuanto a la forma, como ya se ha comentado, lo fundamental para la integración paisajística de la escombrera será intentar reproducir las existentes en el entorno natural circundante. En general habrá que huir de las formas geométricas, evitar dejar aristas y superficies excesiamente planas, y redondear taludes en planta y en alzado para darles una apariencia más natural (los perfiles convexos presentan menos riesgos de erosión que los cóncavos, en caso de no poder modelar un perfil totalmente convexo, ir a perfiles mixtos).

Las terrazas o bermas de gran anchura existentes en algunas escombreras producen un efecto visual negativo por lo que desde el punto de vista estético se desaconseja su uso generalizado. Cuando sean imprescindibles para asegurar la estabilidad y control de la erosión de la escombrera se recomienda que sean lo más estrechas posibles, tengan los bordes redondeados y no estén totalmente paralelas.

Como norma genérica y teniendo en cuenta los restantes objetivos de la restauración, es aconsejable que las pendientes de las escombreras oscilen entre el 16-20\% con bermas no superiores a $10 \mathrm{~m}$ de anchura y ligera pendiente hacia el interior, y separación vertical entre ellas inferior a $15 \mathrm{~m}$. En cualquier caso estas cifras pueden variar, dependiendo del tipo de explotación, los materiales estériles, las condiciones impuestas por el medio y las posibles dedicaciones previstas, como las que aparecen en el Cuadro 1.

\section{CUADRO 1}

\begin{tabular}{|c|c|}
\hline Usos previstos para el suelo & $\begin{array}{c}\text { Pendiente } \\
\text { Media (\%) }\end{array}$ \\
\hline - Cultivos agrícolas intensivos & $<5$ \\
- Cultivos agrícolas extensivos & $5-12$ \\
- Cultivos arbóreos y dehesas & $12-20$ \\
- Utilización forestal & $>20$ \\
\hline
\end{tabular}

También hay que tener en cuenta a la hora del modelado de escombreras la estabilidad y drenaje de las mismas, pues tan importante y a veces más que la mimetización de las escombreras en el entorno, es adecuar el diseño para facilitar un drenaje natural del agua superficial; “Un buen planteamiento puede ser la fijación inicial de las rutas de drenaje más convenientes y proyectar la remodelación del terreno de forma que encaje con dicha red de drenaje" (MACRAE, 1983).

Por esto en el modelado no sólo se deben tener en cuenta las pendientes, sino también las sinuosidades en planta de las laderas del entorno, de manera que las escombreras tiendan también a reproducirlas, conservando incluso la situación relativa de las vaguadas (Figura 3).

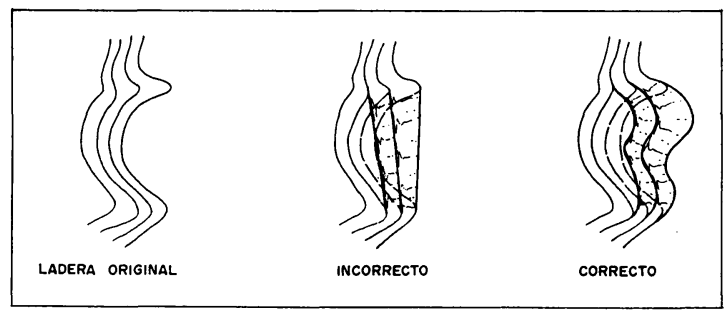

Fig. 3.-Adaptación de las proyecciones en planta de los vertederos a la fisiografía.

En lo referente al diseño los métodos que permiten simultanear las labores de recuperación con las labores de explotación son más ventajosas ya que reducen el impacto global (espaciäl y temporalmente), distribuyen el coste de la restauración a lo largo de la vida de la explotación e incrementan el tiempo disponible para lograr el establecimiento de la vegetación (Figura 4a y b).

Desde el punto de vista paisajístico, el método óptimo de creación de escombreras será aquel que, permitiendo la recuperación progresiva comentada, construya en primer lugar el perímetro exterior de las mismas para luego ir rellenándolas (Figura 5), de forma que las partes exteriores restauradas sirvan de pantalla (visual, sónica y eólica) a los vertidos posteriores.

Debe advertirse que para conseguir la integración paisajística de la escombrera los criterios esbozados de- 


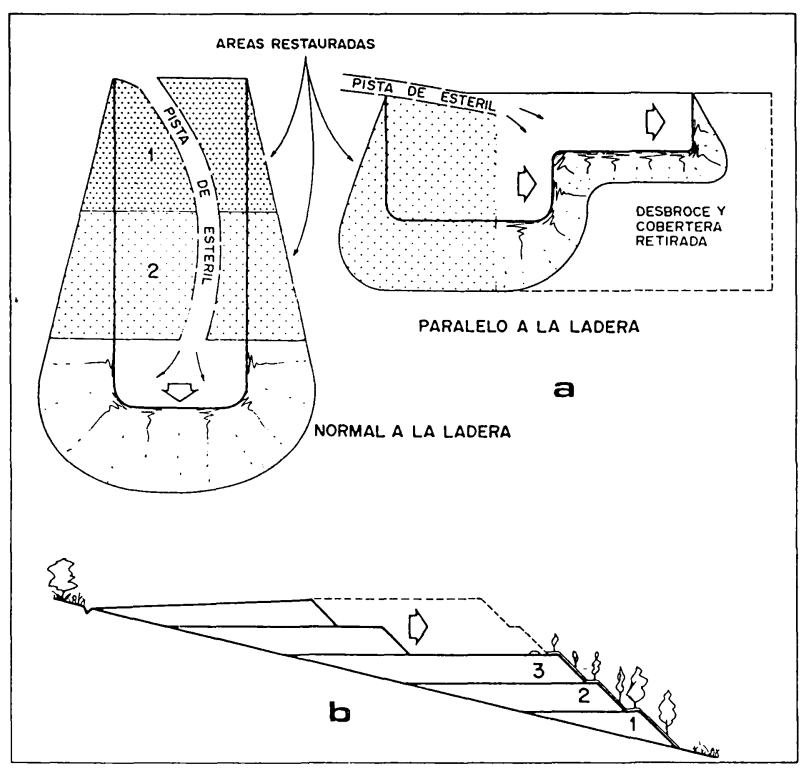

Fig. 4.-Recuperación progresiva de escombreras.

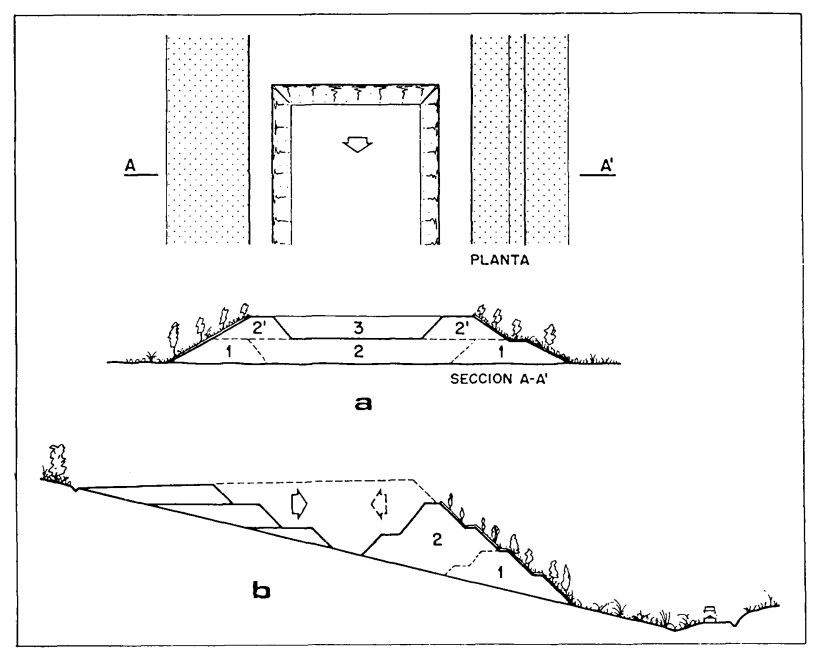

Fig. 5.-Construcción perimetral.

ben complementarse normalmente con el recubrimiento de su superficie con tierra vegetal (contribuye a evitar el contraste cromático que frecuentemente produce el estéril) y posterior introducción de vegetación.

\section{Frentes y taludes finales de corta}

Los elementos y características a considerar para el diseño de los taludes finales de los huecos son básicamente los ya apuntados al hablar de las escombreras. Sin embargo, en este caso las posibilidades de actuación son más limitadas debido, por un lado, a que el emplazamiento es un factor geológico fijo e inamovible, y por otro, a que por las características de los materiales el modelado en general es más difícil.

Aunque no sea la situación más frecuente, habrá ocasiones en que sea posible rellenar el hueco total o par- cialmente, con estériles de la propia explotación o de una cercana, o de utilizarlo como vertedero de residuos de procedencia no minera (en este caso habrá que es. tudiar previamente en profundidad el riesgo de contaminación). Rellenar es la solución que permite acercarse más al estado original del terreno y, por tanto, minimizar el impacto paisajístico, por lo que no debe rèchazarse como alternativa a considerar.

En el caso de las canteras no es frecuente que se originen acopios de escombreras, y el proyecto de recuperación va destinado al enmascaramiento y a la revegetación del frente de corta, que al presentar paredes verticales de naturaleza rocosa y gran dimensión dificultan su recuperación.

Los tratamientos posibles de los taludes finales de las canteras, depende, entre otros factores del tipo y estabilidad del frente y del volumen y naturaleza del material de relleno disponible, como se resumen en la $\mathrm{Fi}$ gura 6 y el Cuadro 2.

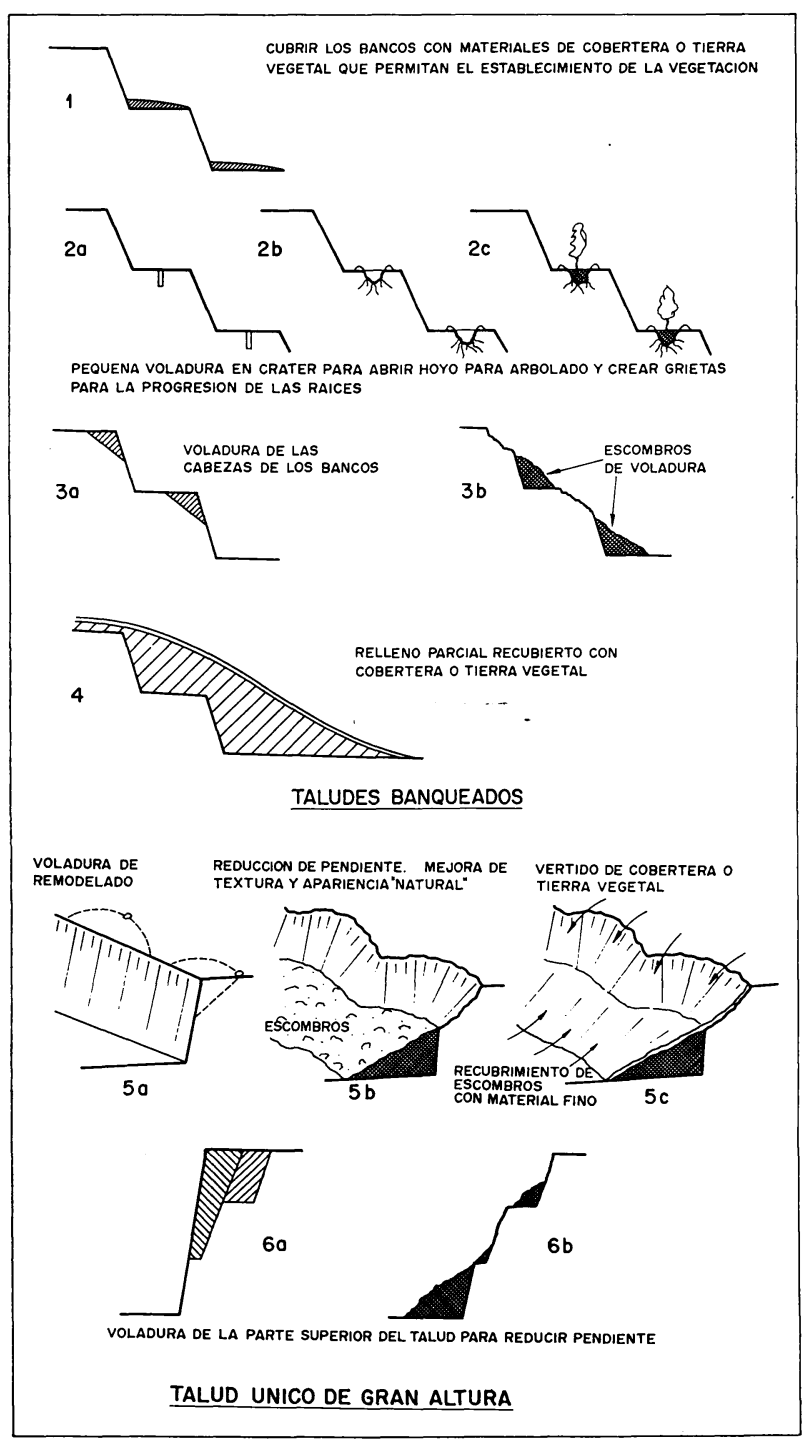

Fig. 6. - Tratamiento de taludes finales de explotación. 
CUADRO 2

Opciones de tratamiento de taludes y huecos (Basado en Coppin y Bradshaw, 1982)

\begin{tabular}{|l|c|c|c|}
\hline \multirow{2}{*}{ OPCION } & \multicolumn{2}{|c|}{ Taludes altos } & Taludes \\
\cline { 2 - 4 } & Único & Banqueado \\
bajos
\end{tabular}

(x) Tratamiento posible.

Los taludes banqueados presentan mayores posibilidades de recuperación que los de frente único; la implantación de arbolado en los bancos ayuda a romper la continuidad del talud y mejora su apariencia. En cualquier caso siempre es aconsejable intentar disminuir la pendiente del talud de corta y dejar pequeñas repisas que favorezcan la acumulación de finos y faciliten el establecimiento de vegetación (Figura 7).

En ocasiones se realizan pequeñas voladuras puntuales con el fin de abrir los hoyos necesarios para la implantación posterior de la vegetación

También se pueden realizar voladuras especiales cuando se desee descabezar los bancos para romper la linealidad y geometría de los taludes (Figura 7).

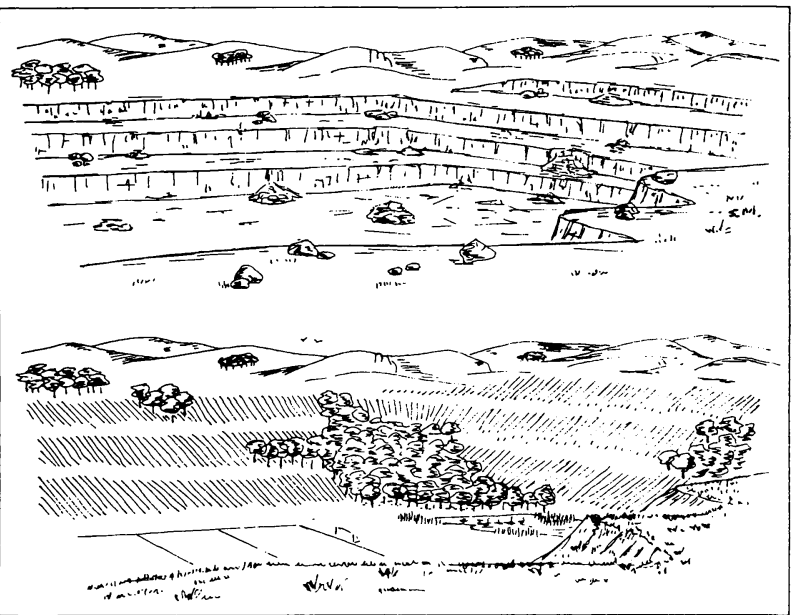

Fig. 7.-Ejemplo de restauración de una cantera, por descabezamiento de los bancos.

(C) Consejo Superior de Investigaciones Científicas

Licencia Creative Commons 3.0 España (by-nc)
En los taludes únicos, de paredes de roca de grandes dimensiones pueden realizarse voladuras controladas con el fin de dar una apariencia más natural al talud al crear pequeñas repisas y disminuir la pendiente. EI talud creado puede recubrirse con cobertera y revegetarse, lo que reducirá notablemente la visibilidad de la pared.

Cuando el contraste cromático producido por el color de la piedra desnuda del talud no pueda ser atenuado por otros métodos (recubrimiento parcial con cobertura, revegetación, etc.), puede estudiarse la posibilidad de aplicar tratamientos especiales de envejecimiento de rocas (fumigación química de la pared, hidrosiembra de paja o mulches y posterior quema, etcétera).

Al igual que en el caso de la creación de escombreras, los frentes y taludes finales de corta en la minería a cielo abierto, pueden restaurarse progresivamente según alcancen su posición final del proyecto, reduciendo la porción visible a los frentes activos. La restauración progresiva permite usar la cobertera (si se hubiera almacenado) o los estériles directamente, sin necesidad de acopiarlos en escombreras.

Conviene resaltar el interés de una planificación "a priori" del diseño modelado tanto de las escombreras como de los taludes finales de corta, para poder conseguir una mayor efectividad y rentabilidad económica. La efectividad de la remodelación planteada después de haberse efectuado la explotación es, desde todo punto de vista, más limitada, ya que no se trata de evitar un impacto, sino de minimizar los ya producidos; esta solución es mucho más difícil y encarece considerablemente el proceso (Figura, pág. siguiente).

\section{RECOMENDACIONES EDÁFICAS}

En general las escombreras presentan unas características poco adecuadas para el desarrollo de la vegetación, la escasa presencia de materia orgánica y la baja proporción de elementos finos. Da lugar a texturas gruesas y a una pobre estabilidad de los agregados arcillo-húmicos, si es que existen. Además hay que tener en cuenta otras características desfavorables pero a la vez muy comunes en este tipo de materiales: valores de $\mathrm{pH}$ extremos, por ejemplo los residuos de carbón tienen $\mathrm{pH}$ muy ácidos, escasa presencia de reservas minerales que da lugar a una débil capacidad de cambio y a una baja disponibilidad de elementos nutritivos (nitrógeno y fósforo principalmente), existencia de elementos tóxicos y sales en concentraciones excesivamente altas para el desarrollo de la vegetación, entre las más significativas.

http://informesdelaconstruccion.revistas.csic.es 


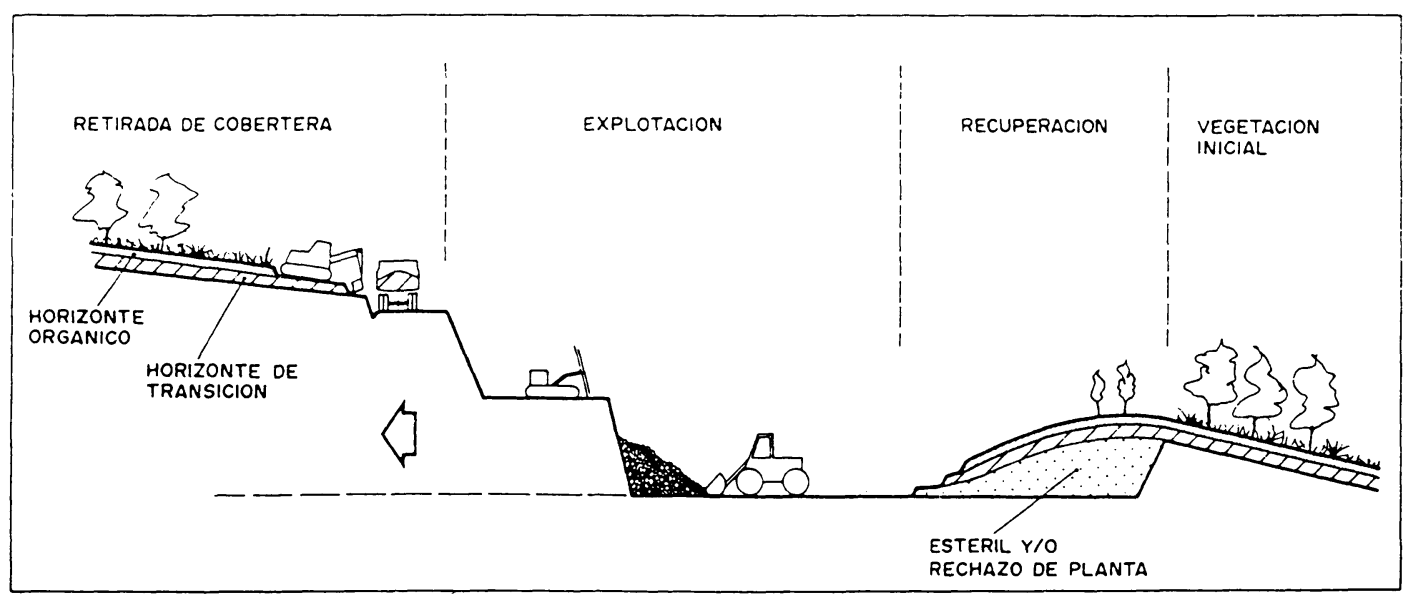

Fig. 8.-Extracción y recuperación progresiva.

Una práctica muy adecuada para la recuperación de las zonas alteradas por la minería es la retirada progresiva y el posterior almacenamiento de las capas superficiales del suelo. Es conveniente agrupar, por separado y en montones no excesivamente grandes, para evitar el deterioro de sus propiedades edáficas, por un lado la cobertera, que corresponde a los primeros centímetros del suelo y que coincide generalmente con los horizontes orgánicos y por otro las capas subyacentes, más pobres en materia orgánica y elementos nutritivos, que se encuentran por encima del yacimiento mineral propiamente dicho.

Si no se ha realizado una planificación de la explotación o bien las condiciones edáficas de la cobertera que ha de extenderse por encima de las escombreras no presenta los mínimos aceptables para que se desarrolle la nueva vegetación a implantar es necesario realizar una serie de enmiendas edáficas encaminadas a mejorar las características de los materiales superficiales que forman las escombreras a recuperar.

Las actuaciones más inmediatas han de dirigirse a proporcionar materia orgánica y elementos nutritivos, ya que si se logra el desarrollo de una cubierta vegetal que pueda irse incorporando al ciclo edáfico, lentamente se comenzará a desarrollar un suelo cada vez más evolucionado. El resto de las actuaciones dependerá del tipo de mineral que se esté explotando. Por ejemplo, será necesario efectuar un encalado con carbonato cálcico o similar en el caso de escombreras procedentes de la minería de carbón para elevar el pH hasta la neutralidad, también puede ser recomendable realizar lavados sucesivos, teniendo en cuenta que no se produzca contaminación en los acuíferos subyacentes. En otros casos (canteras, areneros, minerales no metálicos) será necesario añadir elementos finos como arcillas y sesquióxidos de hierro y aluminio para mejorar las características estructurales de los materiales y fomentar la formación del complejo absorbente del suelo.
El aporte de nutrientes al suelo se puede realizar directamente mediante fertilizantes inorgánicos e indirectamente añadiendo materia orgánica, bien en forma de abonos (purines, estiércol, compost, turba, etc.), o bien extendiendo una capa de tierra vegetal sobre las escombreras.

El nitrógeno es uno de los elementos nutritivos fundamentales para el desarrollo de la vegetación. Los fertilizantes nitrogenados deben añadirse de manera que la planta pueda disponer de nitrógeno a lo largo de todo su ciclo vegetativo. Al comienzo de la siembra sería conveniente añadir nitratos (fácilmente lixiviable si la planta no los asimila rápidamente), para posteriormente ir añadiendo urea, nitrato amónico u otras formas de nitrógeno que contenga a éste en forma de catión amonio de asimilación más lenta y que por lo tanto se pierde menos por percolación. Los fertilizantes de tipo NPK (15-15-15 ó 18-18-18 principalmente) son muy útiles porque además de lo anteriormente expuesto aportan fósforo que es otro nutriente fundamental para la vegetación. La siembra de leguminosas favorece la presencia de nitrógeno en el suelo, al tener la propiedad de fijar en su rizobium el nitrógeno atmosférico.

Las dosis de fertilizantes orgánicos vendrán determinadas por el tipo de escombrera y por la existencia o no de acopio de cobertera. Si no se ha procedido a la retirada de las capas superficiales de suelo natural es muy conveniente realizar un aporte de tierra vegetal de unos 30 a $40 \mathrm{~cm}$ y nunca inferior a $10 \mathrm{~cm}$, dependiendo de la dedicación posterior a la que vaya a ser destinado: los árboles necesitan mayor espesor que los pastizales y cultivos. La tierra vegetal sirve de cama de siembra y como almacén de nutrientes.

Una vez sembradas las especies seleccionadas, es conveniente protegerlas y crear un microclima adecuado para su germinación. Para ello se suelen añadir agentes estabilizadores o mulches que reducen la erosión superficial y protegen las semillas. Estos agentes sue- 
len añadirse en solución acuosa a la mezcla de la hidrosembradora.

\section{ESTABLECIMIENTO DE LA VEGETACIÓN. SELECCIÓN DE ESPECIES VEGETALES}

En la selección de las especies vegetales a tener en cuenta en la recuperación de terrenos alterados por la minería, son muchos los factores y criterios a considerar:

\section{- El clima que prevalece en la zona a recuperar.}

- La naturaleza del sustrato, de la cual depende la capacidad de las distintas especies para vivir en las condiciones de $\mathrm{pH}$, profundidad del suelo, disponibilidad de agua, estructura, temperatura, exigencia de nutrientes, tolerancia a elementos tóxicos, salinidad, etcétera.

- Características y cualidades ecológicas de las especies. Permite contemplar la aptitud de cada una de ellas frente al medio ambiente en el que se va a implantar: grado de protección, velocidad de crecimiento, fenología, altura, longevidad, cubierta, arraigo en el transplante, facilidad de encontrar en vivero, rapidez de germinación, resistencia a plagas, enfermedades, fuegos y competitividad.

- La dedicación o uso principal que se pretenda dar a los terrenos sometidos a explotación minera. $\mathrm{Ca}$ be destacar la recuperación de vegetación natural, el uso agropecuario, el uso forestal y recreativo principalmente.

Para hacer una primera selección de las especies que pueden ser utilizadas en la revegetación, será necesario hacer un análisis de la vegetación potencial y la natural existente en el entorno, esto permite guiar la elección de especies a las mejor adaptadas ecológicamente a la zona, así como de las especies que colonizan de forma espontánea antiguas escombreras, y aquellas especies no autóctonas que aunque no sean propias de este lugar, sus características y cualidades hacen valiosa, y a veces imprescindible su utilización.

A partir de las especies posibles se confecciona una mezcla, como proporción equilibrada de las especies, en la que intervendrán herbáceas, diferenciando gramíneas y leguminosas (de gran labor edificadora en este tipo de sustrato casi estéril) en proporción 2 a 1, y leñosas (matas, arbustos y alguna arbórea).

Los métodos de establecimiento de la vegetación seleccionada son la siembra y la plantación.

La siembra es la forma más barata y rápida de establecer la cubierta vegetal en los terrenos de pendiente inferior a un $25 \%$. Cuando la pendiente supere el $25 \%$ se aplica la técnica de la hidrosiembra que resulta más cara.

La plantación se reserva para la implantación de las especies arbóreas no incluidas en la mezcla indicada, en hoyos previamente cavados.

El éxito de la revegetación también depende de los cuidados posteriores a la implantación, aconsejándose efectuar labores someras, fertilizaciones y riegos adecuados, o incluso el empleo de tratamiento con mulches.

En todo el proceso de la elección de especies no deben olvidarse nunca los objetivos fundamentales de restitución e integración del terreno en su entorno social, ecológico y paisajístico.

\section{REFERENCIAS}

Bradshaw, A. D. and Chadwick, M. J., "The restoration of land", Blackwell, Oxford, 1980.

Coppin, N. J. and Bradshaw, A. D., "Quarry reclamation", Mining Journal Books Ltd., London, 1982.

Macrae, S. G., "The soil scientist's contribution to quarry design and reclamation. Reclamation'83. Industrial Seminars, Tunbridge Wells, 1983.

IGME, "Estudio geoambiental para la restauración del medio natural. Cuenca de El Guadiato, Cuenca de Padul, Madrid, 1987.

IGME, "Programa nacional de estudios geoambientales aplicados a la minería. Provincia de León, Madrid, 1988. 